\title{
$\mathrm{N}^{1}$-甲基鸟嘌呤阳离子脱质子动力学的研究
}

\author{
吴丽丹节家龙刘坤辉* 苏红梅* \\ (中国科学院化学研究所 分子反应动力学国家重点实验室 北京 100190)
}

\begin{abstract}
摘要 在所有 DNA 碱基中, 鸟嘌呤碱基 $\mathrm{G}$ 具有最低的氧化电位, 导致其最容易被氧化. $\mathrm{G}$ 碱基被单电子氧化成为 $\mathrm{G}$ 正 离子自由基 $\left(\mathrm{G}^{+}\right), \mathrm{G}^{+}$存在两个脱质子位点, 其中脱嘧啶环上亚氨基质子 $\mathrm{N}^{1}-\mathrm{H}$ 比脱环外氨基质子 $\mathrm{N}^{2}-\mathrm{H}$ 更有利, 因而在 普通 $\mathrm{G}$ 碱基中研究脱 $\mathrm{N}^{2}-\mathrm{H}$ 的过程无法排除脱 $\mathrm{N}^{1}-\mathrm{H}$ 过程的干扰, 使得其脱 $\mathrm{N}^{2}-\mathrm{H}$ 的动力学迄今尚不明确. 在本文中, 通 过将 $\mathrm{G}$ 碱基上的 $\mathrm{N}^{1}-\mathrm{H}$ 用 $\mathrm{CH}_{3}$ 取代(即 $\mathrm{mG}$ ), 采用纳秒时间分辨瞬态紫外可见吸收光谱方法研究了 $\mathrm{mG}$ 碱基单电子氧化 后脱质子 $\mathrm{N}^{2}-\mathrm{H}$ 的动力学. 根据瞬态紫外可见吸收光谱, 确定了 $\mathrm{mG}^{+*}$ 脱质子的产物是 $\mathrm{mG}\left(\mathrm{N}^{2}-\mathrm{H}\right)^{*}$, 即脱质子的位点是

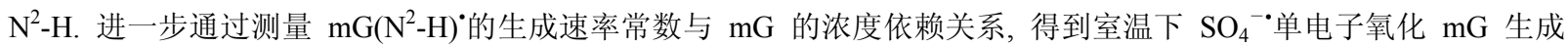

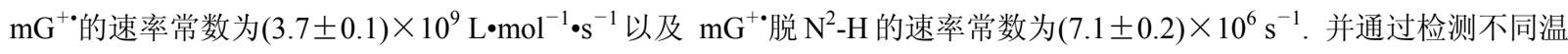
度下 $\mathrm{mG}^{+}$脱 $\mathrm{N}^{2}-\mathrm{H}$ 的速率常数, 利用阿仑尼乌斯方程得出脱质子 $\mathrm{N}^{2}-\mathrm{H}$ 的活化能为 $19.9 \pm 1.0 \mathrm{~kJ} \cdot \mathrm{mol}^{-1}$. 这些结果可为 DNA 碱基的氧化损伤过程提供更为丰富的动力学信息.
\end{abstract}

关键词 $\mathrm{N}_{1}$-甲基鸟嘌呤; 脱质子; 单电子氧化; 速率常数; 活化能

\section{Deprotonation Kinetics of 1-Methylguanine After One-Electron Oxidation}

\author{
Wu, Lidan Jie, Jialong Liu, Kunhui* Su, Hongmei* \\ (State Key Laboratory of Molecular Reaction Dynamics, Beijing National Laboratory for Molecular Sciences (BNLMS), \\ Institute of Chemistry, Chinese Academy of Sciences, Beijing 100190, China)
}

\begin{abstract}
Among the four natural DNA bases, guanine $(\mathrm{G})$ is the most sensitive to oxidation due to its lowest oxidation potential. When $\mathrm{G}$ base is oxidized to guanine cation radical $\left(\mathrm{G}^{+}\right)$, it will deprotonate from both the imino proton $\mathrm{N}^{1}-\mathrm{H}$ and the amino proton $\mathrm{N}^{2}-\mathrm{H}$. According to the $\mathrm{pKa}$ values for $\mathrm{N}^{1}-\mathrm{H}$ and $\mathrm{N}^{2}-\mathrm{H}$ deprotonation, the main deprotonation site in $\mathrm{G}$ base is $\mathrm{N}^{1}-\mathrm{H}$ which would interfere with the $\mathrm{N}^{2}-\mathrm{H}$ deprotonation, making the kinetics of $\mathrm{N}^{2}-\mathrm{H}$ deprotonation difficult to be measured. Herein, the $\mathrm{N}^{2}-\mathrm{H}$ deprotonation kinetics is investigated using 1-methylguanosine $(\mathrm{mG})$, where $\mathrm{N}^{1}-\mathrm{H}$ is substituted by methyl group to avoid the $\mathrm{N}^{1}-\mathrm{H}$ deprotonation and $\mathrm{N}^{9}-\mathrm{H}$ is substituted by ribose to ensure enough solubility of methylguanine in water, by nanosecond transient absorption (ns-TA) spectroscopy. By $355 \mathrm{~nm}$ photolysis of $\mathrm{Na}_{2} \mathrm{~S}_{2} \mathrm{O}_{8}$, the highly oxidizing radical $\mathrm{SO}_{4}^{-}$is generated, which will oxidize $\mathrm{mG}$ to $\mathrm{mG}^{+\bullet}$ instantaneously. The time-resolved absorption spectra obtained for reaction of $\mathrm{mG}$ with $\mathrm{SO}_{4}{ }^{-}$exhibits transient absorptions for $\mathrm{mG}\left(\mathrm{N}^{2}-\mathrm{H}\right)^{\cdot}$ featured by absorption band at $600 \mathrm{~nm}$, indicating that the $\mathrm{mG}^{+\bullet}$ deprotonation product is $\mathrm{mG}\left(\mathrm{N}^{2}-\mathrm{H}\right)^{\cdot}$ and the deprotonation site is therefore validated to be $\mathrm{N}^{2}-\mathrm{H}$. The $\mathrm{mG}$ concentration dependence of $\mathrm{mG}\left(\mathrm{N}^{2}-\mathrm{H}\right)^{\cdot}$ formation rate constant is assessed through changing the $\mathrm{mG}$ concentration from 0.25 $\mathrm{mmol} \cdot \mathrm{L}^{-1}$ to $5 \mathrm{mmol} \cdot \mathrm{L}^{-1}$. The concentration dependence experiment reveals that the rate-limiting step to form $\mathrm{mG}\left(\mathrm{N}^{2}-\mathrm{H}\right)^{\bullet}$ is the bimolecular reaction of $\mathrm{mG}$ with $\mathrm{SO}_{4}{ }^{-}$when $\mathrm{mG}$ concentration is lower than $2 \mathrm{mmol} \cdot \mathrm{L}^{-1}$ and the bimolecular reaction

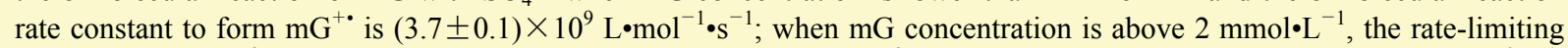
step to form $\mathrm{mG}\left(\mathrm{N}^{2}-\mathrm{H}\right)^{\bullet}$ is the first-order $\mathrm{mG}^{+}$deprotonation and the $\mathrm{N}^{2}-\mathrm{H}$ deprotonation rate constant is $(7.1 \pm 0.2) \times 10^{6} \mathrm{~s}^{-1}$. Furthermore, the $\mathrm{N}^{2}-\mathrm{H}$ deprotonation rate constant is measured at different temperatures varying from $278 \mathrm{~K}$ to $298 \mathrm{~K}$. According to Arrhenius equation, the activation energy barrier for the $\mathrm{N}^{2}-\mathrm{H}$ deprotonation is determined to be $19.9 \pm 1.0 \mathrm{~kJ}$. $\mathrm{mol}^{-1}$. These results can provide valuable kinetic information on the oxidative damage of DNA.
\end{abstract}

Keywords 1-methylguanine; deprotonation; one-electron oxidation; rate constant; activation energy barrier

\section{1 引言}

DNA 是生物体的遗传物质，它的氧化损伤会导致 错误的编码, 引起突变, 癌症和细胞的衰老 ${ }^{[1,2]}$. 这些损 伤可以发生在 DNA 链的各个部位 ${ }^{[3 \sim 5]}$, 包括碱基、戊糖
以及磷酸一戊糖连接处. 其中, DNA 碱基是最容易发生 氧化损伤的 ${ }^{[6]}$, 并且它们的氧化损伤尤其有害, 因为它 会直接导致 DNA 结构的破坏 ${ }^{[7,8]}$. 在四种 DNA 碱基中 (腺嘌呤 $\mathrm{A}$, 鸟嘌呤 $\mathrm{G}$, 胞嘧啶 $\mathrm{C}$ 和胸腺嘧啶 $\mathrm{T}$ ), 鸟嘌呤 $\mathrm{G}$ 由于其氧化电位最低 $\left(E^{o}=1.29 \mathrm{~V} \text { vs NHE }\right)^{[9,10]}$, 因而

*E-mail: khliu@iccas.ac.cn; hongmei@iccas.ac.cn

Received September 28, 2014; published October 22, 2014.

Project supported by the National Natural Science Foundation of China (No. 21333012), the National Basic Research Program of China (No. 2013CB834602), and the Chinese Academy of Sciences (No. XDB12020200).

项目受国家自然科学基金(No. 21333012)、“973”基金(No. 2013CB834602)及中国科学院先导专项(No. XDB12020200)资助. 
最容易被氧化损伤. 当 $\mathrm{G}$ (结构式如图 1 所示)被单电子 氧化形成阳离子自由基 $\mathrm{G}^{+\cdot}$ 后，一般认为随后的过程是 脱 $\mathrm{N}^{1}-\mathrm{H}$ 生成 $\mathrm{G}\left(\mathrm{N}^{1}-\mathrm{H}\right)^{\cdot[11 \sim 13]}$ (本文对 $\mathrm{N}$ 的标识采取图 1 的约定, 分别表示为 $N^{1}, N^{2}$ ), 并且测得 $G^{+}$上 $N^{1}-H$ 的 $\mathrm{pKa}$ 为 $3.9^{[11,13]}$. 然而, 已有的研究表明环外 $\mathrm{N}^{2}-\mathrm{H}$ 上的 $\mathrm{pKa}$ 为 $4.7^{[11,12]}$, 与 $\mathrm{G}^{+\cdot}$ 上 $\mathrm{N}^{1}-\mathrm{H}$ 的 $\mathrm{pKa}(3.9)$ 值相差不大. 理论计算研究发现 $\mathrm{G}^{+\bullet}$ 脱 $\mathrm{N}^{1}-\mathrm{H}$ 产物 $\mathrm{G}\left(\mathrm{N}^{1}-\mathrm{H}\right)$ 和脱 $\mathrm{N}^{2}-\mathrm{H}$ 产物 $\mathrm{G}\left(\mathrm{N}^{2}-\mathrm{H}\right)^{*}$ 的能量相近 ${ }^{[14 \sim 16]}$, 并表明在 $\mathrm{G}$ 中从环外的 氨基 $\mathrm{N}^{2}$ 上脱质子与从嘧啶环上的亚氨基 $\mathrm{N}^{1}$ 上脱质子是 可以竞争的 ${ }^{[17]}$. 此外, 在低温玻璃态溶液 ${ }^{[18]}$ 以及固 体 ${ }^{[19,20]}$ 条件下氧化 $\mathrm{G}$ 碱基的实验中, 确实检测到了产物 $\mathrm{G}\left(\mathrm{N}^{2}-\mathrm{H}\right)^{\cdot}$, 说明在正常的 $\mathrm{G}$ 碱基被氧化后, 确实存在脱 $\mathrm{N}^{2}-\mathrm{H}$ 的通道.

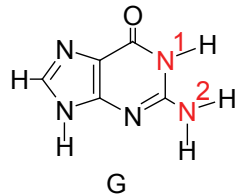

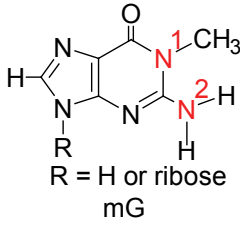

图 $1 \mathrm{G}$ 和 $\mathrm{mG}$ 的分子结构式

Figure 1 The structure of $\mathrm{G}$ and $\mathrm{mG}$

反应速率常数, 活化能等是表征化学反应动力学的 重要参数. 相对于 $\mathrm{G}^{+}$脱质子 $\mathrm{N}^{1}-\mathrm{H}$ 动力学已有大量深入 的研究 ${ }^{[13,21 ~ 24]}, \mathrm{G}^{+}$脱质子 $\mathrm{N}^{2}-\mathrm{H}$ 的动力学却鲜少有报道. 因为普通 $\mathrm{G}$ 碱基被氧化形成 $\mathrm{G}^{+}$后, 由于 $\mathrm{N}^{1}-\mathrm{H}$ 的 $p K a(3.9)^{[11,13]}$ 稍小于 $N^{2}-H$ 上的 $p K a(4.7)^{[11]}$, 脱 $N^{1}-H$ 是 主要的过程, 并会对脱 $\mathrm{N}^{2}-\mathrm{H}$ 通道的研究产生干扰, 使 得脱 $\mathrm{N}^{2}-\mathrm{H}$ 的动力学难以研究. 在本文中, 我们研究了 鸟嘌呤碱基单电子氧化后脱质子 $\mathrm{N}^{2}-\mathrm{H}$ 的速率常数以及 活化能. 为了避免脱 $\mathrm{N}^{1}-\mathrm{H}$ 通道对脱 $\mathrm{N}^{2}-\mathrm{H}$ 过程研究的干 扰，我们选取 $\mathrm{N}^{1}-\mathrm{H}$ 被 $\mathrm{CH}_{3}$ 取代的 $\mathrm{G}$ 碱基 $(\mathrm{mG}$, 结构式如 图 1 所示)作为研究对象. 为了增加 $\mathrm{mG}$ 在水溶液中的溶 解度, 我们实验所用的是带戊糖的 $\mathrm{mG}$ 碱基(以下文中 的 $\mathrm{mG}$ 都指带戊糖的 $\mathrm{mG}$ 碱基), 其中戊糖不会对所要研 究的碱基部分的反应动力学过程产生影响 ${ }^{[11,13]}$. 通过氧 化性非常强的 $\mathrm{SO}_{4}{ }^{-}\left(E^{o}=2.43 \mathrm{~V} \text { vs NHE }\right)^{[25]}$ 氧化 $\mathrm{mG}$ 产 生 $\mathrm{mG}^{+\cdot}$, 采用纳秒时间分辨瞬态紫外可见吸收光谱方 法研究了随后 $\mathrm{mG}^{+}$脱 $\mathrm{N}^{2}-\mathrm{H}$ 的动力学. 通过瞬态紫外可 见吸收光谱, 我们首先确认了 $\mathrm{mG}^{+}$脱质子的位点确实 是 $\mathrm{N}^{2}-\mathrm{H}$. 而通过改变 $\mathrm{mG}$ 的浓度, 得到了 $\mathrm{mG}$ 与 $\mathrm{SO}_{4}{ }^{-}$. 双分子反应生成 $\mathrm{mG}^{+}$的速率常数以及 $\mathrm{mG}^{+}$脱 $\mathrm{N}^{2}-\mathrm{H}$ 的 速率常数. 最后, 通过改变温度测量脱质子 $\mathrm{N}^{2}-\mathrm{H}$ 的速 率常数, 利用阿仑尼乌斯(Arrhenius)方程得到了脱 $\mathrm{N}^{2}-\mathrm{H}$ 的活化能, 为 DNA 碱基的氧化脱质子过程提供了动力 学基本数据.

\section{2 结果与讨论}

\section{$2.1 \mathrm{SO}_{4}{ }^{-*}$ 的产生}

根据文献报道 ${ }^{[26]}$, 采用 $355 \mathrm{~nm}$ 激光照射 $\mathrm{Na}_{2} \mathrm{~S}_{2} \mathrm{O}_{8}$ 溶液，可导致溶液中 $\mathrm{S}_{2} \mathrm{O}_{8}{ }^{2-}$ 的 $\mathrm{O}-\mathrm{O}$ 键发生断裂，生成 $\mathrm{SO}_{4}{ }^{-*}$ (方程 1):

$$
\mathrm{S}_{2} \mathrm{O}_{8}{ }^{2-}+\mathrm{h} v(355 \mathrm{~nm}) \rightarrow 2 \mathrm{SO}_{4}{ }^{-\cdot}
$$

实验中，用 $355 \mathrm{~nm}$ 激光光解 $400 \mathrm{mmol} \cdot \mathrm{L}^{-1}$ 的 $\mathrm{Na}_{2} \mathrm{~S}_{2} \mathrm{O}_{8}$ 水溶液 $(\mathrm{pH}=7.5$ ) 得到的 $50 \mathrm{~ns}$ 时刻的吸收光谱 如图 2 所示. 从图中可看出, 激光照射过后, 溶液在 310 $550 \mathrm{~nm}$ 范围内都有吸收, 最强吸收峰在 $450 \mathrm{~nm}$ 位 置. 这与 Shafirovich 等 ${ }^{[26]}$ 报道的 $\mathrm{SO}_{4}{ }^{-}$吸收光谱一致, 说明光解产生了 $\mathrm{SO}_{4}{ }^{-\bullet} . \mathrm{SO}_{4}{ }^{-\cdot}$ 在 $450 \mathrm{~nm}$ 处的消光系数为

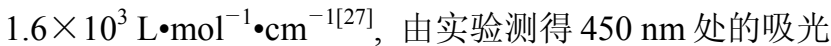
度(A) 可以得到光解产生 $\mathrm{SO}_{4}^{-\cdot}$ 的浓度为 $14 \mu \mathrm{mol} \cdot \mathrm{L}^{-1}$. $\mathrm{S}_{2} \mathrm{O}_{8}{ }^{2-}$ 的光解离过程非常快 $(\sim 14 \mathrm{~ns})^{[26]}$, 因此 $\mathrm{SO}_{4}{ }^{-\cdot}$ 生 成动力学不会对之后的次级反应过程研究造成干扰.

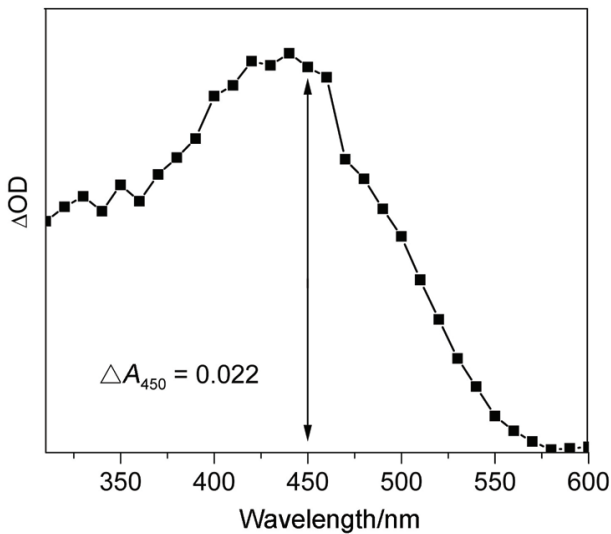

图 $2355 \mathrm{~nm}$ 激光光解 $400 \mathrm{mmol} \cdot \mathrm{L}^{-1}$ 的 $\mathrm{Na}_{2} \mathrm{~S}_{2} \mathrm{O}_{8}$ 水溶液 $(\mathrm{pH}=7.5)$ 在 $50 \mathrm{~ns}$ 时刻的光谱

Figure 2 Transient UV-vis spectra at $50 \mathrm{~ns}$ for buffer solution $(\mathrm{pH}=$ 7.5) of $\mathrm{Na}_{2} \mathrm{~S}_{2} \mathrm{O}_{8}\left(400 \mathrm{mmol} \cdot \mathrm{L}^{-1}\right)$ upon $355 \mathrm{~nm}$ laser excitation

\section{$2.2 \mathrm{mG}$ 中脱质子的位点}

由 $355 \mathrm{~nm}$ 激光瞬时产生的 $\mathrm{SO}_{4}^{-\cdot}$ 可以氧化 $\mathrm{mG}$ 产生

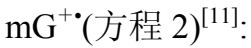

$$
\mathrm{SO}_{4}{ }^{-\cdot}+\mathrm{mG} \rightarrow \mathrm{SO}_{4}{ }^{2-}+\mathrm{mG}^{+\cdot}
$$

因为 $\mathrm{mG}^{+\cdot}$ 的 $\mathrm{pKa}$ 为 $4.7^{[11]}$, 在 $\mathrm{pH}=7.5$ 的条件下会 发生脱质子的反应(方程 3):

$\mathrm{mG}^{+\cdot} \rightleftharpoons \mathrm{mG}\left(\mathrm{N}^{2}-\mathrm{H}\right)^{\cdot}+\mathrm{H}^{+}$

由于 $\mathrm{mG}$ 上的 $\mathrm{N}^{1}-\mathrm{H}$ 已经被 $\mathrm{CH}_{3}$ 取代，因此脱质子 反应只能发生在 $\mathrm{N}^{2}-\mathrm{H}$ 位 ${ }^{[12]}$, 这也可从测定 $\mathrm{mG}$ 与 $\mathrm{SO}_{4}{ }^{-*}$ 的反应产物得到证实. 在室温下, 用 $355 \mathrm{~nm}$ 激光光解 $\mathrm{mG}\left(3 \mathrm{mmol} \cdot \mathrm{L}^{-1}\right)+\mathrm{Na}_{2} \mathrm{~S}_{2} \mathrm{O}_{8}\left(400 \mathrm{mmol} \cdot \mathrm{L}^{-1}\right)$ 的水溶液 $(\mathrm{pH}$ $=7.5)$ 得到瞬态吸收光谱展示在图 3 中. 如图所示, 在 $50 \mathrm{~ns}$ 时刻的吸收光谱上, 在 $400 \mathrm{~nm}$ 和 $500 \mathrm{~nm}$ 有两个吸 收峰, 这与文献中给出的 $\mathrm{mG}^{+}$的吸收峰位置基本一致, 
并且光谱形状也相同 ${ }^{[11,12]}$, 因此 $50 \mathrm{~ns}$ 时刻的吸收光谱 可归属为 $\mathrm{mG}^{+}$. 随着反应的进行, $\mathrm{mG}^{+}$在 $400 \mathrm{~nm}$ 和 500 $\mathrm{nm}$ 处的吸收逐渐衰减, 并伴随着一个 $600 \mathrm{~nm}$ 处新峰的 生成(图 3). 对 $400 \mathrm{~nm}$ 和 $600 \mathrm{~nm}$ 处的谱峰进行单指数动 力学拟合(图 3 插图)得到的时间常数一致 $(\sim 160 \mathrm{~ns})$, 说 明 $400 \mathrm{~nm}$ 光谱的衰减和 $600 \mathrm{~nm}$ 光谱的上升对应于同一 个过程, 即 $\mathrm{mG}^{+} \cdot$ 脱质子. 由文献可知 ${ }^{[11,12,28,29]}$, 理论计 算和实验都表明 $\mathrm{mG}^{+\bullet}$ 脱 $\mathrm{N}^{2}-\mathrm{H}$ 的产物 $\mathrm{mG}\left(\mathrm{N}^{2}-\mathrm{H}\right)^{\bullet}$ 在 600 $\mathrm{nm}$ 附近有吸收峰, 并且 $\mathrm{mG}\left(\mathrm{N}^{2}-\mathrm{H}\right)^{\text {光谱 }}{ }^{[11,12]}$ 与图 3 中 2 $\mu \mathrm{s}$ 时刻的吸收光谱形状相似. 因此 $600 \mathrm{~nm}$ 处的吸收峰 可归属为 $\mathrm{mG}\left(\mathrm{N}^{2}-\mathrm{H}\right)^{*}$. 可见, 由瞬态吸收光谱可以确定 $\mathrm{mG}^{+}$脱质子的产物是 $\mathrm{mG}\left(\mathrm{N}^{2}-\mathrm{H}\right)^{\bullet}, \mathrm{mG}^{+}$脱质子的位点 确实是 $\mathrm{N}^{2}-\mathrm{H}$.

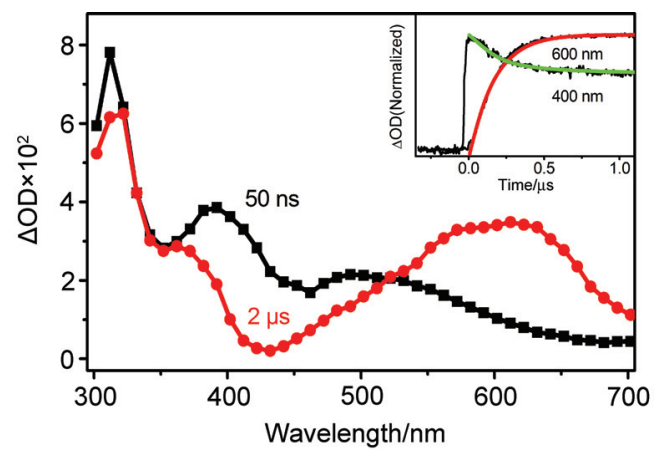

图 $3355 \mathrm{~nm}$ 激光光解 $\mathrm{mG}\left(3 \mathrm{mmol} \cdot \mathrm{L}^{-1}\right)+\mathrm{Na}_{2} \mathrm{~S}_{2} \mathrm{O}_{8}\left(400 \mathrm{mmol} \cdot \mathrm{L}^{-1}\right)$ 水 溶液 $(\mathrm{pH}=7.5)$ 在 $50 \mathrm{~ns}$ (黑色)及 $2 \mu \mathrm{s}$ (红色)时刻的光谱. 插图显示的是 实验得到的 $400 \mathrm{~nm}$ 和 $600 \mathrm{~nm}$ (黑色)的动力学以及对它们的拟合曲线 (分别为绿色和红色)

Figure 3 Transient UV-vis spectra monitored at $50 \mathrm{~ns}$ (black square) and $2 \mu \mathrm{s}$ (red circle) for buffer solution $(\mathrm{pH}=7.5)$ of $\mathrm{mG}\left(3 \mathrm{mmol} \cdot \mathrm{L}^{-1}\right)+$ $\mathrm{Na}_{2} \mathrm{~S}_{2} \mathrm{O}_{8}\left(400 \mathrm{mmol} \cdot \mathrm{L}^{-1}\right)$ upon $355 \mathrm{~nm}$ laser excitation. Inset: kinetics traces for $\mathrm{mG}^{+}$decay at $400 \mathrm{~nm}$ and $\mathrm{mG}\left(\mathrm{N}^{2}-\mathrm{H}\right)^{\cdot}$ growth at $600 \mathrm{~nm}$, with the first-order fit to the data (green and red lines)

\section{$2.3 \mathrm{mG}$ 中脱 $\mathrm{N}^{2}-\mathrm{H}$ 的速率}

图 3 的瞬态吸收光谱表明, $600 \mathrm{~nm}$ 是脱质子后中性 自由基 $\mathrm{mG}\left(\mathrm{N}^{2}-\mathrm{H}\right)^{\circ}$ 的特征吸收峰. 通过对 $600 \mathrm{~nm}$ 处动 力学的上升过程进行拟合, 可以得到生成 $\mathrm{mG}\left(\mathrm{N}^{2}-\mathrm{H}\right)^{\circ}$ 的 速率常数. 然而, $\mathrm{mG}\left(\mathrm{N}^{2}-\mathrm{H}\right)^{\circ}$ 的生成包含氧化(反应 2 )和 脱质子(反应 3) 两个步骤. 在 $\mathrm{mG}$ 浓度较低的情况下, 如 果反应的决速步是 $\mathrm{SO}_{4}{ }^{-\cdot}$ 氧化 $\mathrm{mG}$ (即 $k_{3}>k_{2}[\mathrm{mG}]$ ), 反应 3 中的平衡在氧化过程中就可达到, 脱质子过程(反应 3) 将观测不到. 为了区分氧化和脱质子过程, 我们在室温 下进行了 $\mathrm{mG}\left(\mathrm{N}^{2}-\mathrm{H}\right)$ 的生成速率常数随 $\mathrm{mG}$ 浓度变化的 实验, 得到的结果如图 4 所示. 当 $\mathrm{mG}$ 浓度小于 2 $\mathrm{mmol} \cdot \mathrm{L}^{-1}$ 时, $\mathrm{mG}\left(\mathrm{N}^{2}-\mathrm{H}\right)^{\circ}$ 的生成速率常数与 $\mathrm{mG}$ 的浓度 成线性关系, 这表明此时反应的决速步是 $\mathrm{mG}_{\text {与 }} \mathrm{SO}_{4}{ }^{-} \cdot$ 的双分子反应. 由直线的斜率可以算得 $\mathrm{mG}$ 与 $\mathrm{SO}_{4}^{-}$双 分子反应的二级速率常数为 $(3.7 \pm 0.1) \times 10^{9} \mathrm{~L} \cdot \mathrm{mol}^{-1}$. $\mathrm{s}^{-1}$. 当 $\mathrm{mG}$ 浓度大于 $2 \mathrm{mmol} \cdot \mathrm{L}^{-1}$ 时, $\mathrm{mG}\left(\mathrm{N}^{2}-\mathrm{H}\right)^{\bullet}$ 的生成 速率常数达到最大且不再随浓度变化, 说明此时 $\mathrm{mG}^{+}$.
脱质子过程变成反应的决速步. 平台区 $\mathrm{mG}\left(\mathrm{N}^{2}-\mathrm{H}\right)^{\bullet}$ 生成 的速率常数(图 4 红色线段)即是 $\mathrm{mG}^{+}$脱 $\mathrm{N}^{2}-\mathrm{H}$ 的速率常 数, 为 $(7.2 \pm 0.3) \times 10^{6} \mathrm{~s}^{-1}$.

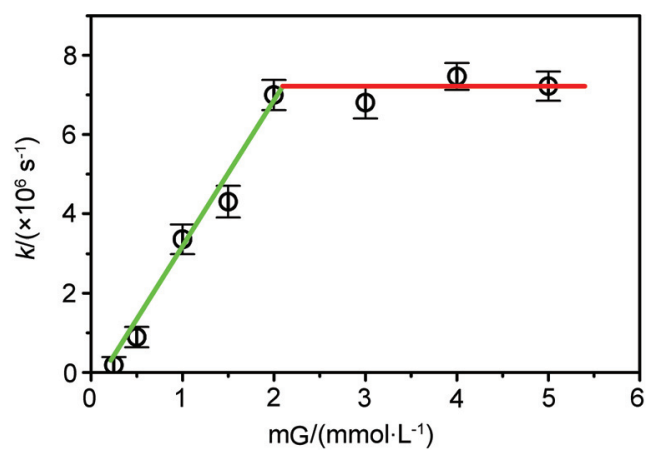

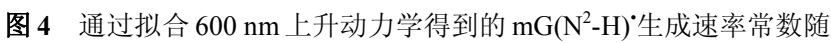
$\mathrm{mG}$ 浓度的变化

Figure $4 \mathrm{mG}$ concentration dependence of $\mathrm{mG}\left(\mathrm{N}^{2}-\mathrm{H}\right)^{\cdot}$ formation rate constant obtained from the increase of the absorbance at $600 \mathrm{~nm}$

根据文献可知, DNA 的 G 碱基被单电子氧化后脱质 子 $\mathrm{N}^{1}-\mathrm{H}$ 的速率常数为 $1.8 \times 10^{7} \mathrm{~s}^{-1[13]}$. 一方面, 我们测 得的 $\mathrm{mG}$ 脱 $\mathrm{N}^{2}-\mathrm{H}$ 的速率常数 $\left(7.2 \times 10^{6} \mathrm{~s}^{-1}\right)$ 比 $\mathrm{G}^{+}$脱 $\mathrm{N}^{1}-\mathrm{H}$ 的速率常数小, 与 $\mathrm{N}^{1}-\mathrm{H}(\mathrm{pKa}=3.9)$ 和 $\mathrm{N}^{2}-\mathrm{H}(\mathrm{pKa}=$ 4.7)的 $\mathrm{pKa}$ 值吻合. 另一方面, $\mathrm{G}^{+}$脱 $\mathrm{N}^{1}-\mathrm{H}$ 的速率常数仅 是脱质子 $\mathrm{N}^{2}-\mathrm{H}$ 的速率常数的 2.5 倍. 因而脱质子 $\mathrm{N}^{2}-\mathrm{H}$ 相对于脱 $\mathrm{N}^{1}-\mathrm{H}$ ，虽然是次要反应通道，但也不能被忽 略. 在常温水溶液中单电子氧化 $\mathrm{G}$ 碱基, Steenken 等 ${ }^{[11]}$ 和 Kobayashi 等 ${ }^{[13]}$ 只观测到脱 $\mathrm{N}^{1}-\mathrm{H}$ 的产物，未能观测到 脱 $\mathrm{N}^{2}-\mathrm{H}$ 产物, 这可能是由于在水溶液中脱 $\mathrm{N}^{1}-\mathrm{H}$ 产物比 脱 $\mathrm{N}^{2}-\mathrm{H}$ 产物稳定, 脱 $\mathrm{N}^{2}-\mathrm{H}$ 产物可以迅速异构化为脱 $\mathrm{N}^{1}-\mathrm{H}$ 产物 ${ }^{[17]}$. 而在低温玻璃态溶液以及固体条件下, 脱 $\mathrm{N}^{2}-\mathrm{H}$ 产物无法快速转化为脱 $\mathrm{N}^{1}-\mathrm{H}$ 产物 ${ }^{[17]}$, 因而可检 测到脱 $\mathrm{N}^{2}-\mathrm{H}$ 产物 ${ }^{[18 ~ 20]}$, 说明脱质子 $\mathrm{N}^{2}-\mathrm{H}$ 产物通道确实 存在. 而在 DNA 链中, 由于 $\mathrm{N}^{1}-\mathrm{H}$ 和 $\mathrm{N}^{2}-\mathrm{H}$ 所处的环境 更复杂, $\mathrm{G}$ 碱基中 $\mathrm{N}^{1}-\mathrm{H}$ 以及 $\mathrm{N}^{2}$ 上的一个氢原子容易形 成氢键，因而 $\mathrm{N}^{2}$ 上另一个自由的氢原子有可能成为主 要的脱质子位点. 作为系列研究, 本课题组正在开展关 于 DNA 单链, 双链以及四连体中 $\mathrm{G}$ 碱基脱质子动力学 (脱质子的位点以及速率常数) 的研究, 本文的研究结果 可为这类 DNA 链中的反应提供有意义的参考.

\section{4 脱质子 $\mathrm{N}^{2}-\mathrm{H}$ 的活化能}

活化能是表征化学反应动力学的一个重要参数, 活 化能越低，化学反应越容易发生，并且反应的速率越快. 为了更好的表征 $\mathrm{mG}$ 中脱 $\mathrm{N}^{2}-\mathrm{H}$ 的动力学过程, 我们在 不同温度下 $(278 \sim 298 \mathrm{~K})$ 测量了 $\mathrm{mG}^{+\bullet}$ 脱 $\mathrm{N}^{2}-\mathrm{H}$ 速率常数, 从而获得 $\mathrm{mG}$ 中脱质子 $\mathrm{N}^{2}-\mathrm{H}$ 的活化能. 实验中使用 $\mathrm{mG}$ 的浓度为 $3 \mathrm{mmol} \cdot \mathrm{L}^{-1}$, 在此浓度下反应决速步为脱质子 过程(如图 4 所示), 由 $600 \mathrm{~nm}$ 吸收的动力学拟合得到的 速率常数对应脱质子速率常数, 而不是氧化速率常数. 
不同温度下测得的 $600 \mathrm{~nm}$ 处光谱的动力学曲线如图 5 所示. 从图 5 中可看出, 随着反应温度的降低, 脱质子 的速率常数逐渐变慢. 进一步通过单指数动力学拟合得 到不同温度下脱质子的速率常数(如表 1 所示). 根据阿 仑尼乌斯方程, 反应速率常数与温度有如下关系(方程 4):

$$
\ln k=\ln A-E_{\mathrm{a}} / R T
$$

$\ln k$ 对 1000/T 作图(如图 6 所示)符合很好的线性关 系, 由直线的斜率可以获得脱质子 $\mathrm{N}^{2}-\mathrm{H}$ 的活化能 $E_{\mathrm{a}}$ 为 $19.9 \pm 1.0 \mathrm{~kJ} \cdot \mathrm{mol}^{-1}$. 由此可知脱质子 $\mathrm{N}^{2}-\mathrm{H}$ 的活化能非 常小, 当 $\mathrm{mG}$ 碱基被氧化后, 脱 $\mathrm{N}^{2}-\mathrm{H}$ 的反应也很容易发 生.

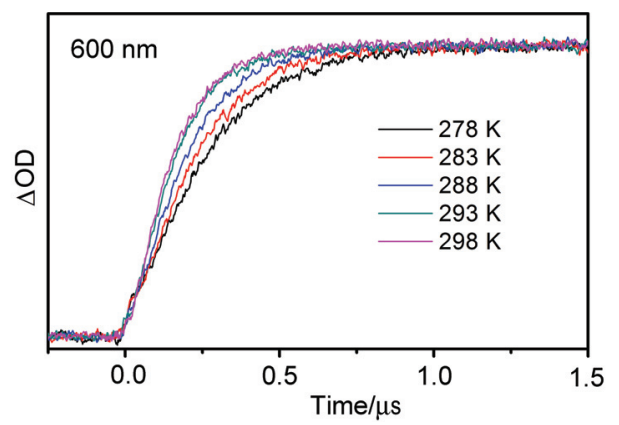

图 5 归一化后不同温度下测得 $600 \mathrm{~nm}$ 处光谱的动力学曲线

Figure 5 Normalized kinetics curves of the absorbance at $600 \mathrm{~nm}$ at different temperatures

表 1 不同温度下的反应时间常数及速率常数

Table 1 Reaction time constants and rate constants obtained at different temperature

\begin{tabular}{lll}
\hline$T / \mathrm{K}$ & $\tau / \mathrm{ns}$ & $k / \times 10^{6} \mathrm{~s}^{-1}$ \\
\hline 298 & $150 \pm 2$ & $6.7 \pm 0.1$ \\
293 & $164 \pm 4$ & $6.1 \pm 0.2$ \\
288 & $194 \pm 3$ & $5.1 \pm 0.1$ \\
283 & $229 \pm 5$ & $4.4 \pm 0.1$ \\
278 & $262 \pm 5$ & $3.8 \pm 0.1$ \\
\hline
\end{tabular}

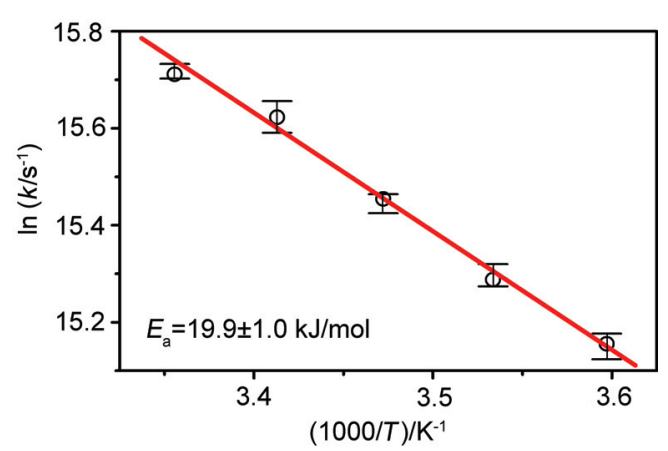

图 6 脱质子速率常数随温度的变化(圆圈所示)及其拟合曲线(红色直 线)

Figure 6 Arrhenius plots of the deprotonation rate constants at temperatures in the range of $278 \sim 298 \mathrm{~K}$ in steps of $5 \mathrm{~K}$, with the activation energy indicated. Solid line is the fitting line

\section{3 结论}

在本文中, 我们采用强氧化性自由基 $\mathrm{SO}_{4}{ }^{-\bullet}$ 氧化 $\mathrm{N}^{1}$ 甲基鸟嘌呤碱基, 利用瞬态吸收光谱研究了 $\mathrm{mG}$ 碱基单 电子氧化后发生脱质子 $\mathrm{N}^{2}-\mathrm{H}$ 的动力学. 通过时间分辨 紫外可见吸收光谱, 我们确认了 $\mathrm{mG}^{+\cdot}$ 脱质子位点是环 外的氨基 $\mathrm{N}^{2}-\mathrm{H}$. 测量不同 $\mathrm{mG}$ 浓度下中性自由基产物 $\mathrm{mG}\left(\mathrm{N}^{2}-\mathrm{H}\right)$ 的生成速率常数, 我们发现当 $\mathrm{mG}$ 浓度低于 2 $\mathrm{mmol} \cdot \mathrm{L}^{-1}$ 时, $\mathrm{mG}$ 与 $\mathrm{SO}_{4}{ }^{-}$的双分子反应是生成 $\mathrm{mG}\left(\mathrm{N}^{2}-\mathrm{H}\right)^{\circ}$ 的决速步, 双分子反应的速率常数为 $(3.7 \pm$

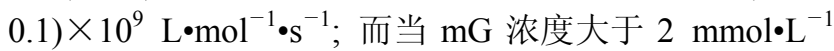
时, $\mathrm{mG}^{+}$脱质子 $\mathrm{N}^{2}-\mathrm{H}$ 成为反应的决速步, $\mathrm{mG}^{+\bullet}$ 脱 $\mathrm{N}^{2}-\mathrm{H}$ 的速率常数为 $(7.1 \pm 0.2) \times 10^{6} \mathrm{~s}^{-1}$. 进一步, 在不同温度 下测量 $\mathrm{mG}^{+} \cdot$ 脱 $\mathrm{N}^{2}-\mathrm{H}$ 的速率常数, 由阿仑尼乌斯方程得 到脱 $\mathrm{N}^{2}-\mathrm{H}$ 的活化能为 $19.9 \pm 1.0 \mathrm{~kJ} \cdot \mathrm{mol}^{-1}$. 在实验中, 我们将 $\mathrm{mG}$ 被氧化生成 $\mathrm{mG}^{+}$的过程与 $\mathrm{mG}^{+}$脱 $\mathrm{N}^{2}-\mathrm{H}$ 的 过程很好地区分开, 分别得到 $\mathrm{mG}^{+}$的生成速率常数与 脱质子速率常数, 同时还获得了脱质子过程的活化能, 为 DNA 碱基的氧化损伤过程提供了更为丰富的动力学 信息.

\section{4 实验部分}

\section{1 不同温度下样品的制备}

首先将 $\mathrm{mG}(\mathrm{BERRY})$ 与 $\mathrm{Na}_{2} \mathrm{~S}_{2} \mathrm{O}_{8}$ (Sigma) 溶于 50 $\mathrm{mmol} \cdot \mathrm{L}^{-1}$ 的 $\mathrm{pH}=7.5$ 的磷酸缓冲溶液(北京索莱宝)中, $\mathrm{mG}$ 与 $\mathrm{Na}_{2} \mathrm{~S}_{2} \mathrm{O}_{8}$ 的浓度分别为 $3 \mathrm{mmol} \cdot \mathrm{L}^{-1}$ 和 $400 \mathrm{mmol} \cdot$ $\mathrm{L}^{-1}$. 取出 $3 \mathrm{~mL}$ 溶液放入 $1 \mathrm{~cm} \times 1 \mathrm{~cm}$ 的石英比色㿼中, 再将比色具放入液氮低温恒温器(牛津仪器 Optistat DN) 的样品仓中, 通过温度控制器设定实验所用的温度, 恒 温 $1 \mathrm{~h}$, 以确保样品达到实验所需的温度.

\section{2 激光闪光光解}

$\mathrm{mG}$ 与 $\mathrm{SO}_{4}^{-}$反应的瞬态紫外可见吸收光谱以及反 应的动力学曲线由 Edinburgh LP920 型闪光光解仪测得. 实验中, 采用 Continuum Surelite II 型 Nd:YAG 激光器作 为激发光源. 由半高全宽为 $7 \mathrm{~ns}$ 的 $355 \mathrm{~nm}$ 脉冲激光激 发样品(频率设定为 $10 \mathrm{~Hz}$ ). 检测光源为 $450 \mathrm{~W}$ 的脉冲 氙灯。单色仪和光电倍增管(R928, Hamamatsu)组成的 检测系统采集 $300 \mathrm{~nm}$ 到 $700 \mathrm{~nm}$ 范围的紫外可见瞬态吸 收光谱. 由光电倍增管输出的随时间变化的信号, 在 $100 \mathrm{MHz}$ 的示波器(TDS 3012C, Tektronix)上显示并记 录, 然后数据传送到计算机进行处理并储存. 采集到的 数据用 LP920 光谱仪自带的软件进行处理. 为了确保实 验结果准确可信, 实验数据都是重复三次取平均值.

\section{References}

[1] Lapi, A.; Pratviel, G.; Meunier, B. Met.-Based Drugs 2001, 8, 47.

[2] Zhang, Q. H.; Wang, Y.; Liu, C.; Yang, Z. Z. Acta Chim. Sinica 2014, 72, 956. (张千慧, 王阳, 刘翠, 杨忠志, 化学学报, 2014, 72, 956.) 
[3] Yin, W. F.; Ou, Z. Z.; Gao, Y. Y.; Hao, P.; Guo, C. L.; Wang, Z. L. Acta Chim. Sinica 2010, 68, 1343. (殷卫峰, 欧植泽, 高云燕, 郝 平, 郭创龙, 王中丽, 化学学报, 2010,68, 1343.)

[4] Wang, X. X.; Gu, Y.; Chen, D. X.; Fang, Y. F.; Huang, Y. P. Acta Chim. Sinica 2010, 68, 2463. (王晓星, 顾彦, 陈登霞, 方艳芬, 黄 应平，化学学报, 2010, 68,2463 .)

[5] Lu, Y. M.; Ou, Z. B.; Hu, W.; Le, X. Y. Acta Chim. Sinica 2012, 70 , 973. (卢艳梅, 区志镔, 胡伟, 乐学义, 化学学报, 2012, 70, 973.)

[6] Buxton, G. V.; Greenstock, C. L.; Helman, W. P.; Ross, A. B. J. Phys. Chem. Ref. Data 1988, 17, 513.

[7] Misiaszek, R.; Crean, C.; Joffe, A.; Geacintov, N. E.; Shafirovich, V. J. Biol. Chem. 2004, 279, 32106.

[8] Jaruga, P.; Dizdaroglu, M. Nucleic Acids Res. 1996, 24, 1389.

[9] Steenken, S.; Jovanovic, S. V.; Bietti, M.; Bernhard, K. J. Am. Chem. Soc. 2000, 122, 2373.

[10] Nguyen, K. V.; Burrows, C. J. Acc. Chem. Res. 2012, 45, 2151.

[11] Candeias, L.; Steenken, S. J. Am. Chem. Soc. 1989, 111, 1094.

[12] Candeias, L.; Steenken, S. J. Am. Chem. Soc. 1992, 114, 699.

[13] Kobayashi, K.; Tagawa, S. J. Am. Chem. Soc. 2003, 125, 10213.

[14] Mundy, C. J.; Colvin, M. E.; Quong, A. A. J. Phys. Chem. A 2002, 106, 10063 .

[15] Wetmore, S. D.; Boyd, R. J.; Eriksson, L. A. J. Phys. Chem. B 1998, $102,9332$.

[16] Gervasio, F. L.; Laio, A.; Iannuzzi, M.; Parrinello, M. Chem.-Eur. J. 2004, 10, 4846 .
[17] Adhikary, A.; Kumar, A.; Becker, D.; Sevilla, M. D. J. Phys. Chem. $B$ 2006, 110, 24171.

[18] Adhikary, A.; Kumar, A.; Munafo, S. A.; Khanduri, D.; Sevilla, M. D. Phys. Chem. Chem. Phys. 2010, 12, 5353.

[19] Close, D. M.; Sagstuen, E.; Nelson, W. H. J. Chem. Phys. 1985, 82, 4386.

[20] Hole, E. O.; Sagstuen, E.; Nelson, W. H.; Close, D. M. Radiat. Res. 1992, $129,1$.

[21] Kobayashi, K.; Yamagami, R.; Tagawa, S. J. Phys. Chem. B 2008, $112,10752$.

[22] Kumar, A.; Sevilla, M. D. J. Phys. Chem. B 2009, 113, 11359.

[23] Liu, N.; Ban, F. Q.; Boyd, R. J. J. Phys. Chem. A 2006, 110, 9908.

[24] Ceron-Carrasco, J. P.; Requena, A.; Perpete, E. A.; Michaux, C.; Jacquemin, D. J. Phys. Chem. B 2010, 114, 13439.

[25] Huie, R. E.; Clifton, C. L.; Neta, P. Radiat. Phys. Chem. 1991, 38 477.

[26] Rokhlenko, Y.; Geacintov, N. E.; Shafirovich, V. J. Am. Chem. Soc 2012, 134, 4955

[27] Mcelroy, W. J. J. Phys. Chem. 1990, 94, 2435.

[28] Chatgilialoglu, C.; Caminal, C.; Guerra, M.; Mulazzani, Q. G. Angew. Chem., Int. Ed. 2005, 44, 6030.

[29] Chatgilialoglu, C.; D'Angelantonio, M.; Guerra, M.; Kaloudis, P.; Mulazzani, Q. G. Angew. Chem., Int. Ed. 2009, 48, 2214.

(Cheng, F.) 